\title{
Location-Price Competition in Mobile Operator Market
}

Vladimir Mazalov, Andrey Lukyanenko and Andrei Gurtov

The self-archived postprint version of this journal article is available at Linköping University Institutional Repository (DiVA):

http://urn.kb.se/resolve?urn=urn:nbn:se:liu:diva-1644.3.5

N.B.: When citing this work, cite the original publication.

Mazalov, V., Lukyanenko, A., Gurtov, A., (2019), Location-Price Competition in Mobile Operator Market, International Game Theory Review, 21(3), 1850015.

https://doi.org/10.1142/So219198918500159

Original publication available at:

https://doi.org/10.1142/S0219198918500159

Copyright: World Scientific Publishing

http://www.worldscientific.com/ 


\title{
Location-Price Competition in Mobile Operator Market
}

\author{
Vladimir Mazalov
}

Andrey Lukyanenko

Andrei Gurtov

September 19, 2018

1

\begin{abstract}
In the paper we propose a game-theoretic model of the mobile network market. The market is presented by three sides: primary mobile network operators (MNO), mobile virtual network operators (MVNO) and consumers of the services. MVNO are mobile operators without their own infrastructure. They buy resources from MNO and compete with other MVNO for the consumers selling a service in the mobile network market. We construct a two-stage game. On the first stage MVNO (players) select the MNO, one or several, and then announce the price for their service for the consumers in this MNO. After the profile of prices is determined, the consumers are distributed among MVNOs following the logistic function. The equilibrium in this two-stage game is constructed. For identical consumers the analytic formulas for the solution are derived.
\end{abstract}

\section{Introduction}

Technological maturation leads to market's formation, expansion and segmentation. Moreover, it results in enhancements of services. Indeed, it is true for many modern applications, the few of them are cloud computing, mobile technologies, personal computer architectures. For all these cases, we historically observed how big players create new markets and later small and medium sized enterprises (SMEs) populate it with own unique services and find specific market niche. For example, lately, there are many internet of things (IoT) vendors and various third-party cloud provider services. Currently, 4G mobile networks are widely deployed, and the 5th generation is coming at year 2020 .

This paper constructs a two-level market model for mobile operators and study their behavior in market organization. Namely, we talk about mobile network operators (MNOs) and mobile virtual network operators (MVNO). For these two types of participants we construct a two-level market model and analyze it. Having said that, we emphasize that our model is general-purpose

\footnotetext{
${ }^{1}$ V. Mazalov is at Institute of Applied Mathematical Research, Karelian Research Center, Russian Academy of Science, Pushkinskaya St. 11 and School of Mathematcis and Statistics and Institute of Applied Mathematcis, Qingdao University, Qingdao 266071, PR China; A. Likyanenko is at Department of Computer Science and Engineering, Aalto University, Finland; A. Gurtov is at Department of Computer and Information Science, Linköping University, Sweden
} 
and applicable to many other cases including the aforementioned examples of cloud-computing, hardware (IoT and PC) manufacture and many others.

Worldwide, there are about one thousand MVNOs and three hundred MNOs. One of the leading countries in the number of MVNOs is Germany with over 120 MVNOs. In some countries such as Finland, the number of operators is much smaller, two-three MNOs (Sonera, Elisa, DNA) and a few MVNOs(Saunalahti, Cubio) [3].

The model that we construct is a two-level market model, however we intrinsically consider the third level of final consumers of the market products. In our model, the consumers are incorporated using a special construction - a logistic function - which properties we explain in upcoming section.

The main contributions in this paper are:

1. Formulation of two-level competitive market for MNOs and MVNOs.

2. Finding market organization for identical players.

3. General analysis of the pricing game.

4. Finding equilibrium for allocation games in two-player cases

The rest of the paper is organized as following. In Section 2, we describe the concept of mobile operator markets. In Section 3, we formulate a game-theoretic model of two-stage competition. In Section 4, we start by developing a simple model for identical players. In Section 5, the pricing game is developed and in Section 6 the allocation game. Section 7 summarizes the related work. Finally, Section 8 concludes the paper.

\section{Mobile Network Operator Market}

Mobile network operator (MNO) is an operator of an infrastructure that provides many mobile network services. Normally, it consists of many network components, including cables, mobile stations or base stations, billing, and etc. Nowadays, MNO starts to enrich infrastructure with powerful Cloudlet services $^{2}$. The advance of technology leads to the fact that more sophisticated services are provided by MNOs for end customers and business. The technologies that play the most important role today in these advancements are cloud computing, virtualization, network function virtualization and mobile service offloading.

Flexibility of MNO services result in formation of mobile virtual network operators (MVNOs) - service providers that as MNO provide mobile network services to the end users, but do not own any physical infrastructure (or their infrastructure plays minor role in the business). In a sense, MVNOs are brokers that buy services from MNOs, enhance them and sell to own customers as shown in Figure 1. From customer point of view, the difference between MNOs and MVNOs is negligible. From MNO's point of view, MVNOs are large customers that buy crude services as a wholesale commodity. From MVNO's side, they buy services from MNOs, integrate own product (services) into MNO services and sell the enrich and unique product to customers.

\footnotetext{
${ }^{2}$ https://networks.nokia.com/solutions/multi-access-edge-computing
} 


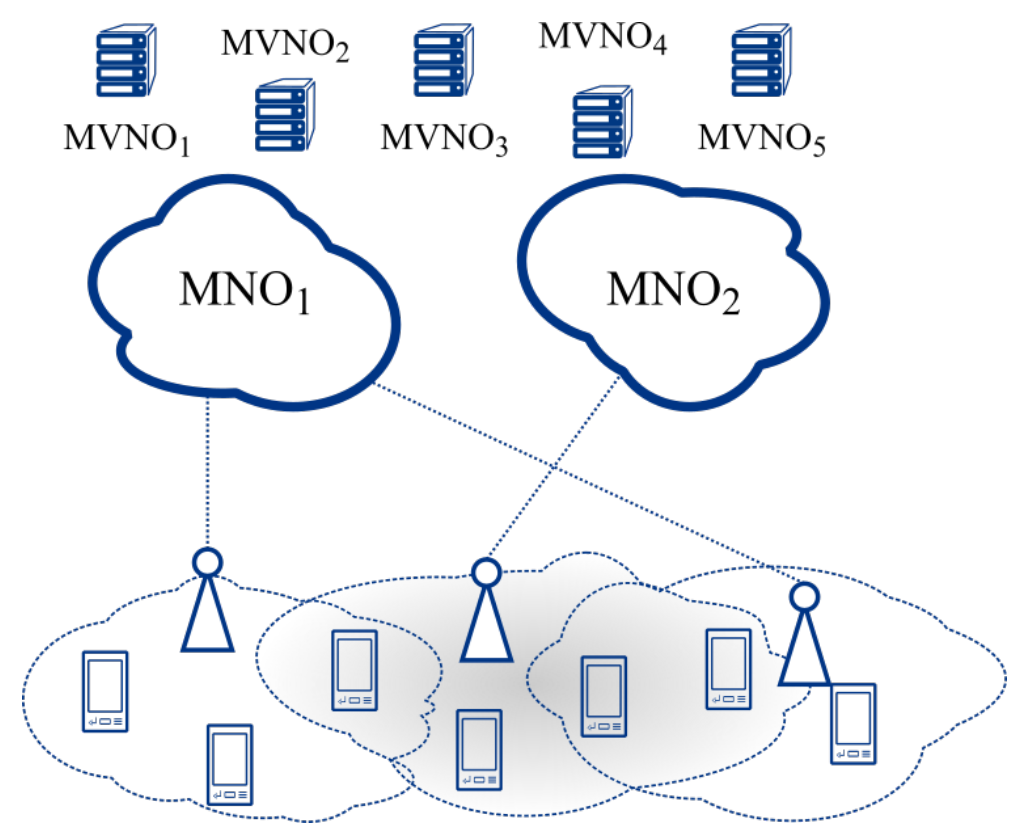

Figure 1: MNO own the infrastructure, while MVNO can choose which MNO to use.

At a first glance, it may appear that MNOs will always dominate over MVNOs, but it turns out that MVNO could efficiently create unique services and increase market revenue. Moreover, MNO normally cannot push MVNOs out of the market due to competition legislation. If MNO wants to create a competitive service to that of MVNO, then the MNO compete on the same level as the MVNO, independently of physical infrastructure. Due to this argumentation, in this paper, we do not consider competition between MNOs an MVNOs, as they occupy different levels of the market organization. Instead, if a MNO wants to start to compete with some MVNO, then this MNO "creates" artificial MVNO on the level of other MVNOs and compete with them on the same level. If real scenario cannot be reduced to this MNO split, then that indicated that MNO is violating competition laws.

Each user select MVNO services based on a set of factors, such as brand name, flexibility, price, availability, uniqueness and locality - the list can be long. In order to deal with user preference we incorporate a logistic function of a form

$$
\gamma_{i}=\frac{\exp \left\{-\sum_{f \in F} k_{i f} r_{i f}\right\}}{\sum_{j \in I} \exp \left\{-\sum_{f \in F} k_{j f} r_{j f}\right\}}, \forall i \in I,
$$

where $I$ is the set of all MVNOs and $\gamma_{i}$ is the fraction of users that is attracted by MVNO $i$; the interest of customers to each MVNO is measured as a scalar weight value, which is computed as a production of weights - exponents of each influence factor, namely $\exp \left\{-k_{i f} r_{i f}\right\}$ for factor $f$ in set $F$. Each $\gamma_{i}$ is normalized so that $\sum_{i} \gamma_{i}=1$. The logistic function is a natural construction that defines a portion of market attracted by each agent. As we can see, all 
factors are incorporated into the logistic function allowing to deal with as many factors as we want, for each factor we need to provide its "coefficient of influence" - $k_{i f}$, which in many cases is independent of MVNO (i.e., $k_{i f}=k_{f} \forall i$, and "amount of resource" - $r_{i f}$ - a value indicating how much of particular factor parameter is developed for particular MVNO. For example, if we consider a brand-name as a factor, then high $k_{f}$ compared to other coefficients may indicate that users consider brand recognition to be very important, and low $r_{i f}$ that MVNO $i$ did not put a lot of money into brand development and is not so well recognized on the market.

In many cases, some factors are dominating and others (minor parameters) can be omitted. In that case we can skip unnecessary parameters. Additionally, in this paper we study a game where price is a controllable parameter, while brand or some other resource is not controllable, thus instead of writing factors that do not change during the game as a constant multiplier, i.e., $\exp \left\{-\sum_{f \in F} k_{i f} r_{i f}\right\}=K_{i} \exp \left\{-\alpha_{i j} q_{i}\right\}$, where $K_{i}$ is some coefficient for player $i$ and $q_{i}$ is the price for the resources.

Finally, let's discuss market geographical distribution. We know that the market geographical distribution is defined solely by MNO's presence. If there are no MNO in some areas, then there is no possibility to get customers from this area. Moreover, some MNO could operate in one area while other in another, e.g. operator $A$ is only in city $X$, and operator $B$ is only in city $Y$. Each MVNO attracts customers from different regions and areas and if MVNO wants customers in city $Y$, then it has to work with operator $B$. In the most generic and complex case, we need to construct coverage maps of each MNO for each area and construct a graph-based model for the game. However, for simplicity (and we believe without loss of generality), we consider that each MNO has a fixed set of end-users that it covers and MVNOs are competing for the users on these markets defined by MNO coverage. For each resource that MVNOs occupy at market defined by MNO, they pay amount $p$.

\section{Two-Stage Competition Model}

Now let's build a MNO-MVNO market model. There are $m$ mobile network operators (MNO) $\left\{M_{1}, M_{2}, \ldots, M_{m}\right\} . \quad M_{j}$ is characterized by the parameters $\left(p_{j}, m_{j}, r_{j}, c_{j}\right), j=1, \ldots, m$, where $p_{j}$ - price for resource, $m_{j}$ is the number of consumers, $r_{j}$ is amount of a resource and $c_{j}$ is a fee to join to this market. Denote $M=\left\{M_{1}, M_{2}, \ldots, M_{m}\right\}$ the set of operators.

There are $n$ mobile virtual network operators (MVNO) $\left\{V_{1}, V_{2}, \ldots, V_{n}\right\}$ who compete in the market for the resources. We suppose that $n$ is much larger than $m$. Each MVNO $V_{i}$ has some private resource $v_{i}, i=1, \ldots, n$, represented as a scalar number. MVNO are the players in the following game. Competition consists of two stages. On the first stage the players (MVNO) choose some MNO to compete for the consumers. So, the strategy of player $V_{i}$ is a subset $s_{i} \subset S_{i}$ from the feasible set $S_{i} \subseteq M$.

After the profile $s=\left(s_{1}, \ldots, s_{n}\right)$ is formed the players announce the prices for their service in each market $j: q_{i}^{j}, i=1, \ldots, n ; j \in s_{i}$. The profile of the prices we denote $q=\left(q_{1}, \ldots, q_{n}\right)$. To avoid the monopoly we suppose that if the market $M_{j}$ is occupied only by one player $V_{i}$ there is a restriction for the price $q_{i} \leq Q_{j}$. So, the payoff of the player $V_{i}$ on the non-competitive market $M_{j} \in s_{i}$ is equal 
to

$$
u_{i}^{j}(q)=\left(Q_{j}-p_{j}\right) m_{j}-c_{j} .
$$

The payoff of player $V_{i}$ on the competitive market $M_{j} \in s_{i}$ is equal to

$$
u_{i}^{j}(q)=\left(q_{i}^{j}-p_{j}\right) m_{j} \gamma_{i}^{j}-c_{j},
$$

where $\gamma_{i}^{j}$ is a proportion of consumers $m_{j}$ who are interested in the service $V_{i}$. Here we will use the logistic function [6]

$$
\gamma_{i}^{j}=\frac{\exp \left\{-\alpha_{i j} q_{i}+\beta_{i j} v_{i}\right\}}{\sum_{l: j \in s_{l}} \exp \left\{-\alpha_{l j} q_{l}+\beta_{l j} v_{l}\right\}}=\frac{k_{i j} \exp \left\{-\alpha_{i j} q_{i}\right\}}{\sum_{l: j \in s_{l}} k_{l j} \exp \left\{-\alpha_{l j} q_{l}\right\}}, \quad i=1, \ldots, n, j \in s_{i},
$$

where $\alpha_{i j}$ and $\beta_{i j}$ reflect the influence of prices and attractiveness of service $i$ on the number of customers for this service on market $M_{j}$, and

$$
k_{i j}=\exp \left\{\beta_{i j} v_{i}\right\}, \quad i=1, \ldots, n, j \in s_{i}
$$

The general payoff of player $V_{i}$ is the sum of payoffs in all used markets:

$$
\begin{gathered}
u_{i}(q)=\sum_{j \in s_{i}} u_{i}^{j}(q)=\sum_{j \in s_{i}^{\prime}}\left(\left(q_{i}^{j}-p_{j}\right) m_{j} \gamma_{i}^{j}-c_{j}\right)+\sum_{j \in s_{i}^{\prime \prime}}\left(\left(Q_{j}-p_{j}\right) m_{j}-c_{j}\right), \\
i=1, \ldots, n,
\end{gathered}
$$

where $s_{i}^{\prime}$ and $s_{i}^{\prime \prime}$ are competitive and non-competitive markets, respectively.

The objective of this paper is to find an equilibrium in the pricing model and then in the allocation problem.

\section{Model with Identical Players}

At the beginning consider a simple case where all MVNOs are identical, so all parameters $v_{i}, \alpha_{i j}, \beta_{i j}$ do not depend on the player $i$, and all markets are competitive. In this case let us assume $\alpha_{i j}=\alpha_{j}, j=1, \ldots, m$ and $\beta_{i j}=\beta_{j}, j=$ $1, \ldots, m$. For simplicity, assume that $k_{i j}=1$. Consider the pricing game on the first market. Let $n_{1} \geq 2$ players compete in this market. They announce the prices $q=\left(q_{1}, \ldots, q_{n_{1}}\right)$. The payoff of player $V_{i}$ is

$$
u_{i}^{1}(q)=\left(q_{i}-p_{1}\right) m_{1} \frac{\exp \left\{-\alpha_{1} q_{i}\right\}}{\sum_{l=1}^{n_{1}} \exp \left\{-\alpha_{1} q_{l}\right\}}-c_{1}, i=1, \ldots, n_{1} .
$$

The first order condition for the equilibrium $\partial u_{i}(q) / \partial q_{i}=0$ gives

$$
\sum_{l=1}^{n_{1}} \exp \left\{-\alpha_{1} q_{l}\right\}=\left(q_{i}-p_{1}\right) \sum_{l \neq i} \exp \left\{-\alpha_{1} q_{l}\right\} \alpha_{1}
$$

By symmetry all prices in the equilibrium must be equal, for example $q_{1}$. It yields 


$$
q_{1}=p_{1}+\frac{1}{\alpha_{1}} \frac{n_{1}}{n_{1}-1} .
$$

Hence, the optimal payoff of player $V_{i}$ on the first market is

$$
u_{i}^{1}=\frac{m_{1}}{\alpha_{1}} \frac{1}{n_{1}-1}-c_{1}, i=1, \ldots, n_{1} .
$$

We see that the payoff of any player is a decreasing function of the number of players acted in this market. So, the allocation game presented here is a congestion game [10] which has a potential.

If the player $V_{i}$ uses the allocation strategy $s_{i}$ then its general payoff is

$$
u_{i}=\sum_{j \in s_{i}}\left(\frac{m_{j}}{\alpha_{j}} \frac{1}{n_{j}(s)-1}-c_{j}\right), i=1, \ldots, n,
$$

where $n_{j}(s)$ is the number of players choosing the market $M_{j}$ (so called congestion vector). To find the equilibrium in the congestion game we maximize the potential function which has the following form

$$
P\left(s_{1}, \ldots, s_{n}\right)=\sum_{j=1}^{m} \sum_{i=1}^{n_{j}(s)}\left(\frac{m_{j} / \alpha_{j}}{i-1}-c_{j}\right) .
$$

Consequently, the optimal allocation of the players among $\left\{M_{1}, \ldots, M_{m}\right\}$ can be found as a solution of the optimization problem

$$
\sum_{j=1}^{m}\left(\frac{m_{j}}{\alpha_{j}} \sum_{i=1}^{n_{j}} \frac{1}{i-1}-c_{j} n_{j}\right) \rightarrow \max
$$

in condition

$$
\sum_{j=1}^{m} n_{j}=\sum_{i=1}^{n}\left|s_{i}\right|
$$

For example if the players can choose only one market then $\sum_{i=1}^{n}\left|s_{i}\right|=n$. For large $n$ and small fee $\left(c_{j} \approx 0\right)$ the optimization problem becomes

$$
\sum_{j=1}^{m} \frac{m_{j}}{\alpha_{j}} \log n_{j} \rightarrow \max
$$

the solution of which is

$$
n_{j} \approx \frac{\frac{m_{j}}{\alpha_{j}}}{\sum_{l=1}^{m} \frac{m_{l}}{\alpha_{l}}} n, j=1, \ldots, m,
$$

Theorem 1. For large $n$ the players are allocated proportionally to the ratio of numbers of consumers and the weight of the player in the market.

Notice that we do not know the precise location of the player but we know how many players will be in each market.

The result we have obtained here is non-trivial. As all the players are identical the properties of each market (MNO) become the dominating criteria. For 
example, coefficients of price attractiveness for $M V N O_{j}$ toward market $M N O_{i}$ $\left(\alpha_{i j}\right)$ reduces to attractiveness of market and as a result all players will be distributed over markets proportional to ratio of number of consumers on the market to the price attractiveness of the market.

\section{Pricing Game}

Now we consider a general case. Assume that the players are distributed among $M_{j}$ in correspondence with the allocation rule $s=\left(s_{1}, \ldots, s_{n}\right)$. Consider a market $M_{j}$. In this market we have $n_{j}=n_{j}(s)$ players. For convenience let us reenumerate players inside the market from 1 to $n_{j}$. Pricing game in the market $M_{j}$ in fact is a non-cooperative game on $n_{j}$ players with payoff functions

$$
u_{i}^{j}(q)=\left(q_{i}^{j}-p_{j}\right) m_{j} \frac{k_{i j} \exp \left\{-\alpha_{i j} q_{i}\right\}}{\sum_{l: j \in s_{l}} k_{l j} \exp \left\{-\alpha_{l j} q_{l}\right\}}-c_{j}, i=1, \ldots, n_{j} .
$$

In the paper [6] we show that the game with these payoffs is a potential game $([8,5])$ and the equilibrium $q^{*}=\left(q_{1}^{*}, \ldots, q_{n_{j}}^{*}\right)$ in this game can be found as a maximum of potential function

$$
P_{j}(q)=\prod_{i=1}^{n_{j}}\left(q_{i}^{j}-p_{j}\right) \frac{\exp \left\{-\sum_{l=1}^{n_{j}} \alpha_{l j} q_{l}\right\}}{\sum_{l=1}^{n_{j}} k_{l j} \exp \left\{-\alpha_{l j} q_{l}\right\}} .
$$

The equilibrium $q^{*}$ can be found from the first order condition $\partial P_{j}(q) / \partial q_{i}=$ $0, i=1, \ldots, n_{j}$. It yields

$$
\sum_{l=1}^{n_{j}} k_{l j} \exp \left\{-\alpha_{l j} q_{l}^{*}\right\}=\alpha_{i j}\left(q_{i}^{*}-p_{j}\right) \sum_{l=1(l \neq i)}^{n_{j}} k_{l j} \exp \left\{-\alpha_{l j} q_{l}^{*}\right\}, i=1, \ldots, n_{j} .
$$

Suppose that a new player $n_{j}+1$ joins the market $M_{j}$. New equilibrium prices $q^{\prime}=\left(q_{1}^{\prime}, \ldots, q_{n_{j}}^{\prime}, q_{n_{j}+1}^{\prime}\right)$ satisfy the system of equations

$$
\sum_{l=1}^{n_{j}+1} k_{l j} \exp \left\{-\alpha_{l j} q_{l}^{\prime}\right\}=\alpha_{i j}\left(q_{i}^{\prime}-p_{j}\right) \sum_{l=1(l \neq i)}^{n_{j}+1} k_{l j} \exp \left\{-\alpha_{l j} q_{l}^{\prime}\right\}, i=1, \ldots, n_{j}+1
$$

The equations $(6)$ for $i=1, \ldots, n_{j}$ can be rewritten as

$$
\begin{gathered}
\sum_{l=1}^{n_{j}} k_{l j} \exp \left\{-\alpha_{l j} q_{l}^{\prime}\right\}=\alpha_{i j}\left(q_{i}^{\prime}-p_{j}\right) \sum_{l=1(l \neq i)}^{n_{j}} k_{l j} \exp \left\{-\alpha_{l j} q_{l}^{\prime}\right\} \\
+k_{n_{j}+1, j}\left(\alpha_{n_{j}+1, j}\left(q_{n_{j}+1}^{\prime}-p\right)-1\right) \exp \left\{-\alpha_{n_{j}+1, j} q_{n_{j}+1}^{\prime}\right\}, i=1, \ldots, n_{j} .
\end{gathered}
$$

Assume that we consider only the prices for the service satisfying the conditions 


$$
q_{i} \geq p_{j}+\frac{1}{\alpha_{i j}}, \forall i, j
$$

Compare the equations (6) and (8). The left part of the equations is a decreasing function of $q_{i}$ but the right part of it is an increasing function. The difference is only in the additional term in the second line in (8) which is positive. From here it follows that

$$
q_{i}^{\prime} \leq q_{i}^{*}, i=1, \ldots, n_{j} .
$$

Hence, if a new player joins the market the equilibrium prices of the players who compete in the market before it become less.

Moreover, $q^{*}$ is the maximal point of the potential function (5), so

$$
P\left(q^{\prime}\right) \leq P\left(q^{*}\right) .
$$

Consequently, $\partial P(q) / \partial q_{i} \geq 0, \forall i$ for $q=q^{\prime}$. Because the game is potential, the signs of the derivatives of the payoff functions and the potential are the same. So, $\partial u_{i}(q) / \partial q_{i} \geq 0, \forall i$ for $q=q^{\prime}$. It yields the following proposition.

Theorem 2. If a new player joins the market the payoffs of the players who competed in the market before become less.

\section{Allocation Game}

Note that the optimal payoffs of the players in the equilibrium in market $M_{j}$ depend not only on the number of players $n_{j}$, but also depend on the characteristic of the players (parameters $\alpha_{i j}, k_{i j}$ ). Thus, in a general case the allocation game is not potential. It was shown that if the payoffs are player specific in each market and depend only on the number of players in it then the game admits an equilibrium in pure strategies [7]. But here we have a more complicated allocation game.

\subsection{Allocation game of two players in two markets}

Depending on the parameters of the model the equilibrium in the allocation game can achieved in pure and mixed strategies. Consider the simplest case of two players $V_{1}, V_{2}$ and two markets $M_{1}, M_{2}$. For simplicity suppose that the registration fee is $c_{j}=0, j=1,2$.

In case when $V_{1}, V_{2}$ choose $M_{1}$ their payoffs are

$$
u_{i}\left(q_{1}, q_{2}\right)=\left(q_{i}-p_{1}\right) \frac{k_{i 1} \exp \left\{-\alpha_{i 1} q_{1}\right\}}{k_{11} \exp \left\{-\alpha_{11} k_{11}\right\}+k_{21} \exp \left\{-\alpha_{21} q_{2}\right\}}, i=1,2 .
$$

Finding the equilibrium $\left(q_{1}^{*}, q_{2}^{*}\right)$ we obtain that this point lies on the hyperbola

$$
\left(\left(q_{1}^{*}-p_{1}\right) \alpha_{11}-1\right)\left(\left(q_{2}^{*}-p_{2}\right) \alpha_{21}-1\right)=1,
$$




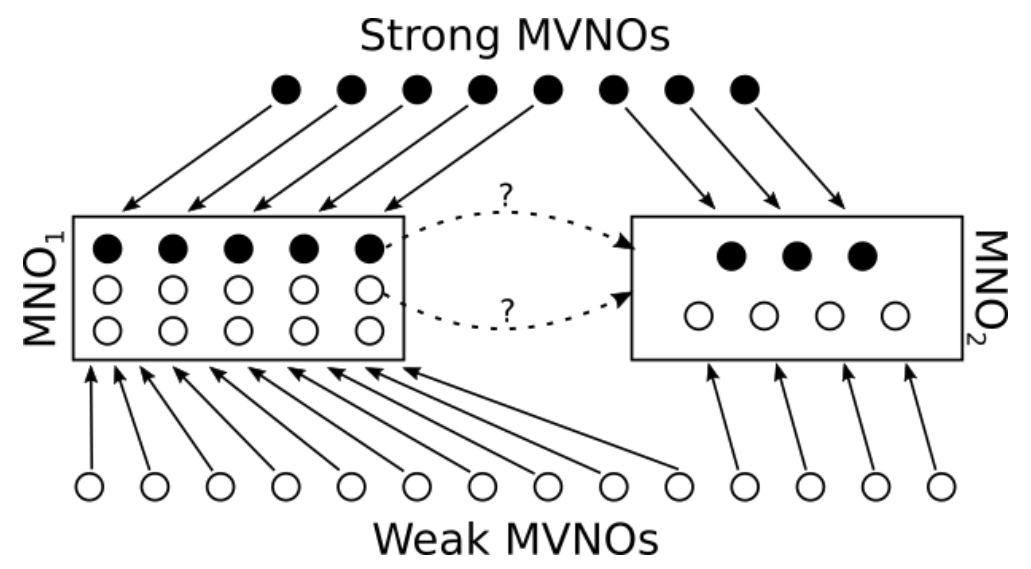

Figure 2: Numeric example of a game for 8 strong and 14 weak MVNOs

and the equilibrium prices satisfy the condition

$$
q_{i}^{*} \geq p_{1}+\frac{1}{\alpha_{i 1}}, i=1,2 .
$$

For symmetric case we showed in Section 2 that the equilibrium prices are equal to

$$
q_{1}^{*}=q_{2}^{*}=p_{1}+\frac{2}{\alpha_{1}}
$$

and the optimal payoffs are

$$
u_{1}^{11}=u_{2}^{11}=\frac{m_{1}}{\alpha_{1}}
$$

In case when $V_{1}, V_{2}$ choose $M_{2}$ their payoffs are

$$
u_{1}^{22}=u_{2}^{22}=\frac{m_{2}}{\alpha_{2}}
$$

In case when $V_{1}, V_{2}$ choose $M_{1}, M_{2}$ respectively then their payoffs are

$$
u_{i}^{12}=\left(Q_{i}-p_{i}\right) m_{i}, i=1,2 .
$$

Finally, if $V_{1}, V_{2}$ choose $M_{2}, M_{1}$ respectively then their payoffs are

$$
u_{i}^{21}=\left(Q_{j}-p_{j}\right) m_{j}, i=1,2 ., i \neq j .
$$

So, we obtain the bimatrix game with the payoff matrix

$$
\left[\begin{array}{cc}
\left(\frac{m_{1}}{\alpha_{1}}, \frac{m_{1}}{\alpha_{1}}\right) & \left(\left(Q_{1}-p_{1}\right) m_{1},\left(Q_{2}-p_{2}\right) m_{2}\right) \\
\left(\left(Q_{2}-p_{2}\right) m_{2},\left(Q_{1}-p_{1}\right) m_{1}\right) & \left(\frac{m_{2}}{\alpha_{2}}, \frac{m_{2}}{\alpha_{2}}\right)
\end{array}\right]
$$

For small $Q_{2}: Q_{2} \leq p_{2}+\frac{m_{1}}{m_{2} \alpha_{1}}$ the equilibrium is to compete for players in the first market, and for small $Q_{1}: Q_{1} \leq p_{1}+\frac{m_{2}}{m_{1} \alpha_{2}}$ the equilibrium is to compete for players in the second market. Otherwise, it is profitable to use different markets. 


\subsection{Stable allocation of identical players in two markets}

Here we consider a problem of stable allocation of $n$ identical players between markets $M_{1}$ and $M_{2}$. Suppose that the market $M-1$ is more attractable than $M_{2}$, i.e.

$$
\frac{m_{1}}{\alpha_{1}} \geq \frac{m_{2}}{\alpha_{2}}
$$

Consider the case when $n_{1} \geq 2$ identical players compete on the market $M_{1}$ and $n_{2} \geq 2$ players compete on the market $M_{2}, n_{1}+n_{2}=n$.

This allocation will be stable if for any player it is not profitable to deviate from the market. Consider market $M_{1}$. Here the payoff of a player $V_{1}$ is

$$
u_{1}^{1}=\frac{m_{1}}{\alpha_{1}\left(n_{1}-1\right)} .
$$

If it moves to market $M_{2}$ its payoff will be

$$
u_{1}^{2}=\frac{m_{2}}{\alpha_{2} n_{2}} .
$$

In case

$$
\frac{m_{1}}{\alpha_{1}\left(n_{1}-1\right)} \geq \frac{m_{2}}{\alpha_{2} n_{2}}
$$

a player from market $M_{1}$ is not interested in the market $M_{2}$. Arguing the same way if

$$
\frac{m_{2}}{\alpha_{2}\left(n_{2}-1\right)} \geq \frac{m_{1}}{\alpha_{1} n_{1}}
$$

the players from market $M_{2}$ are not interested in the market $M_{1}$.

We conclude that a stable allocation takes place for

$$
\frac{\frac{m_{1}}{\alpha_{1}}}{\frac{m_{1}}{\alpha_{1}}+\frac{m_{2}}{\alpha_{2}}}(n-1) \leq n_{1} \leq \frac{\frac{m_{1}}{\alpha_{1}}}{\frac{m_{1}}{\alpha_{1}}+\frac{m_{2}}{\alpha_{2}}}(n-1)+1 .
$$

That is the same allocation as in Section 2 where we suppose that in each market we have at least two players. But it is possible that it is profitable for players to allocate the such way where $n-1$ players act on the market $M_{1}$ and one player acts on the market $M_{2}$.

In Section 5 for two players we show that sometimes it is profitable to choose different markets.

Consider here the case when the stable allocation is such that $n-1$ players compete in the first market and player $M_{n}$ occupies the second market.

In case when the players $V_{1}, \ldots, V_{n-1}$ compete in $M_{1}$ then the equilibrium prices are

$$
q_{i}^{*}=p_{1}+\frac{n-1}{\alpha_{1}(n-2)}, i=1, \ldots, n-1,
$$

and the optimal payoffs are

$$
u_{i}^{1}=\frac{m_{1}}{\alpha_{1}(n-2)}, i=1, \ldots, n-2 .
$$

The payoff of player $n$ is

$$
u_{n}^{2}=\left(Q_{2}-p_{2}\right) m_{2} \text {. }
$$


If player $V_{n}$ joins the first market its payoff will be

$$
u_{n}^{1}=\frac{m_{1}}{\alpha_{1}(n-1)} \text {. }
$$

It is less than $u_{n}^{2}$ if

$$
Q_{2} \geq p_{2}+\frac{m_{1}}{m_{2} \alpha_{1}(n-1)} .
$$

So, in case of (9) player $V_{n}$ will stay in the market $M_{2}$.

Assume that one of players $V_{1}, \ldots, V_{n-1}$ decides to change the current market $M_{1}$ for market $M_{2}$. In this case its payoff will be

It is unprofitable if

$$
u_{1}^{2}=\frac{m_{2}}{\alpha_{2}} .
$$

$$
\frac{m_{2}}{\alpha_{2}} \leq \frac{m_{1}}{\alpha_{1}(n-2)} .
$$

We have a stable allocation if both conditions (9), (10) are satisfied. Collecting together we obtain the condition for the monopoly in one of markets

$$
1+\frac{m_{1}}{\alpha_{1}} \frac{1}{m_{2}\left(Q_{2}-p_{2}\right)} \leq n \leq 2+\frac{\frac{m_{1}}{\alpha_{1}}}{\frac{m_{2}}{\alpha_{2}}} .
$$

We see that monopoly case takes place for a small number of players and for $n>2+\frac{m_{1}}{\alpha_{1}} / \frac{m_{2}}{\alpha_{2}}$ the stable allocation yields the competition in all markets.

\subsection{Stable allocation of non-identical players}

In above we assume that the parameters of players which show the affect of changing of the price on the number of consumers $\alpha_{i j}$ depend on the market but the players are identical. Here we demonstrate the stable allocation of nonidentical MVNOs among MNOs. As now the market share $M_{j}$ for service $i$ depends on the service itself, let us assume $\alpha_{i j}=\alpha^{i}, i=1, \ldots, n$.

Suppose that at the market there are two types of MVNOs. For example, there are "strong" and "weak" mobile virtual operators. Formally, it corresponds to the parameters $\alpha_{i j}=\alpha^{1}$, or $\alpha_{i j}=\alpha^{2}$, for all $j=1, \ldots, m$, and $\alpha^{1} \leq \alpha^{2}$.

Suppose that there are only two markets $M_{1}$ and $M_{2}$ and $n=n_{1}+n_{2}$ mobile virtual operators where $n_{1}, n_{2}$ is the number of "strong" and "weak" MVNO. First of all, the players select a market. Then they play in "pricing game".

Assume that the players are distributed in the following manner. On the market $M_{1}$ the distribution of "strong" and "weak" players is $\left(k_{1}, k_{2}\right)$, and on the market $M_{2}$ the distribution is $\left(l_{1}, l_{2}\right), k_{1}+l_{1}=n_{1}$ and $k_{2}+l_{2}=n_{2}$. Let us find the equilibrium prices in each market. For simplicity assume that all parameters $k_{i j}=1, \forall i, j$.

Consider the market $M_{1}$. the price of MNO here is $p_{1}$. The profile of prices of MVNO is divided for two parts $q=\left(q_{1}^{1}, \ldots, q_{k_{1}}^{1} ; q_{1}^{2}, \ldots, q_{k_{2}}^{2}\right)$, corresponding to "strong" and "weak players", and the payoffs are

$$
u_{i}^{1}(q)=\left(q_{i}^{1}-p_{1}\right) m_{1} \frac{\exp \left\{-\alpha^{1} q_{i}^{1}\right\}}{\sum_{l=1}^{k_{1}} \exp \left\{-\alpha^{1} q_{l}^{1}\right\}+\sum_{l=1}^{k_{2}} \exp \left\{-\alpha^{2} q_{l}^{2}\right\}}-c_{1}, i=1, \ldots, k_{1},
$$


for "strong" players and

$$
u_{i}^{2}(q)=\left(q_{i}^{2}-p_{1}\right) m_{1} \frac{\exp \left\{-\alpha^{2} q_{i}^{2}\right\}}{\sum_{l=1}^{k_{1}} \exp \left\{-\alpha^{1} q_{l}^{1}\right\}+\sum_{l=1}^{k_{2}} \exp \left\{-\alpha^{2} q_{l}^{2}\right\}}-c_{2}, i=1, \ldots, k_{2},
$$

for "weak" players.

The first order condition for the equilibrium $\partial u_{i}^{j}(q) / \partial q_{i}=0, \forall i, j=1,2$, and symmetry of players inside the groups yields that the equilibrium prices for "strong" and "weak players" are equal to $q_{1}^{*}, q_{2}^{*}$, respectively and satisfy the system of equation

$$
\begin{aligned}
& \left(q_{1}-p_{1}\right) \alpha^{1}\left(\left(k_{1}-1\right) \exp \left(-\alpha^{1} q_{1}\right)+k_{2} \exp \left(-\alpha^{2} q_{2}\right)\right)=k_{1} \exp \left(-\alpha^{1} q_{1}\right)+k_{2} \exp \left(-\alpha^{2} q_{2}\right) \\
& \left(q_{2}-p_{1}\right) \alpha^{2}\left(k_{1} \exp \left(-\alpha^{1} q_{1}\right)+\left(k_{2}-1\right) \exp \left(-\alpha^{2} q_{2}\right)\right)=k_{1} \exp \left(-\alpha^{1} q_{1}\right)+k_{2} \exp \left(-\alpha^{2} q_{2}\right) .
\end{aligned}
$$

Hence, the optimal payoff of "strong" player on the first market is

$u^{1}\left(k_{1}, k_{2}, m_{1}\right)=\left(q_{1}^{*}-p_{1}\right) \frac{m_{1}}{\alpha^{1}} \cdot \frac{\exp \left\{-\alpha^{1} q_{1}^{*}\right\}}{\left(k_{1}-1\right) \exp \left\{-\alpha^{1} q_{1}^{*}\right\}+k_{2} \exp \left\{-\alpha^{2} q_{2}^{*}\right\}}-c_{1}, i=1, \ldots, k_{1}$,

and

$u^{2}\left(k_{1}, k_{2}, m_{1}\right)=\left(q_{2}^{*}-p_{1}\right) \frac{m_{1}}{\alpha^{2}} \cdot \frac{\exp \left\{-\alpha^{2} q_{2}^{*}\right\}}{k_{1} \exp \left\{-\alpha^{1} q_{1}^{*}\right\}+\left(k_{2}-1\right) \exp \left\{-\alpha^{2} q_{2}^{*}\right\}}-c_{2}, i=1, \ldots, k_{2}$,

for "weak" players.

The same arguments are true for the equilibrium prices at the market $M_{2}$. Now we can determine when the allocation of $n$ MVNOs among two markets $M_{1}$ and $M_{2}$ will be stable. Allocation $\left[\left(k_{1}, k_{2}\right) ;\left(l_{1}, l_{2}\right)\right]$ is Nash-stable if for each player it is not-profitable to deviate from the current market. Formally, it means that the following inequalities must be satisfied

$$
\begin{gathered}
u^{1}\left(k_{1}, k_{2}, m_{1}\right) \geq u^{1}\left(l_{1}+1, l_{2}, m_{2}\right), \quad u^{2}\left(k_{1}, k_{2}, m_{1}\right) \geq u^{2}\left(l_{1}, l_{2}+1, m_{2}\right), \\
u^{1}\left(k_{1}+1, k_{2}, m_{1}\right) \leq u^{1}\left(l_{1}, l_{2}, m_{2}\right), \quad u^{2}\left(k_{1}, k_{2}+1, m_{1}\right) \leq u^{2}\left(l_{1}, l_{2}, m_{2}\right) .
\end{gathered}
$$

\subsection{Numerical example}

Consider the mobile network market with two MNOs, see Figure 2. The first market is large $m_{1}=1000$, the second is twice smaller $m_{2}=500$. There are twenty two MVNOs competing for the consumers at these markets. Suppose that among these MVNOs there are $n_{1}=8$ "strong" players and $n_{2}=14$ "weak" players, and $\alpha^{1}=1, \alpha^{2}=2$. Let the prices for the resource in both markets be equal $p_{1}=p_{2}=1$, and the costs are $c_{1}=5, c_{2}=2$. Let us show that the allocation

$\left(k_{1}=5, k_{2}=10\right),\left(l_{1}=3, l_{2}=4\right)$ is Nash-stable.

From the equations (11) we find the equilibrium prices in both markets. On the market $M_{1}$ 


$$
q_{1}^{*}=2.125, q_{2}^{*}=1.523 .
$$

The payoffs of the both types players in the equilibrium on the market $M_{1}$ are

$$
u^{1}(5,10,1000)=120.299, u^{2}(5,10,1000)=21.191 .
$$

On the market $M_{2}$ we find

$$
q_{1}^{*}=2.267, q_{2}^{*}=1.550 .
$$

The payoffs of the both types players in the equilibrium on the market $M_{2}$ are

$$
u^{1}(3,4,500)=128.755, u^{2}(3,4,500)=23.242 .
$$

We see that the market $M_{2}$ is more profitable for both types of players. Prove the conditions (12). Suppose, that a "strong" player from the market $M_{1}$ decides to move to the market $M_{2}$. We find that its payoff here is $u^{1}(4,4,500)=102.598$. It is less than its payoff on the market $M_{1}$. So, it is not reasonable to move to another market. Now assume that the "weak player" moves from market $M_{1}$ to the market $M_{2}$. Its payoff here is $u^{2}(3,5,500)=20.700$. It is less than on the market $M_{1}$. So, we see that the allocation $\left(k_{1}=5, k_{2}=10\right),\left(l_{1}=3, l_{2}=4\right)$ is Nash-stable.

\section{Related Work}

Today mobile virtual network operators (MVNO) play an important role in service diversification. However, alone without proper regulatory rules MVNO would not be able to operate, since mobile network operators (MNO) can easily push them out of market [3]. In this paper we do not consider the competition between MVNO and MNO as such, but rather assume that the latter one, in order to compete for the market share, needs to organize a separate child company which will compete for the market with MVNO. Thus, similarly to [6], we consider only the case when end-users select MVNOs based on preferences (such as price, Service Level Agreement (SLA), etc) and MVNOs compete with each other while trying to maximize own revenue and reduce the operational costs. The problem is therefore reduced to a classical setup for the game theory, in a similar way as it was done in [1].

Game theory has a long history and finds its roots in economy where market players compete with each for resources [5]. Since then, the game theory paved its way to other relevant fields in which competition between strategic players appears. Recently the game theoretic approach found its way in cloud computing $[1,6]$. Here multiple brokers compete with each other for users who make decisions which provider to use based on price for computational resources. As indicated in [6], the price is not the only parameter in utility function of an enduser: SLA [1], energy consumption and added value services can be introduced in calculation of Nash equilibrium (NE), too.

To the best of our knowledge the scenario which we consider in this paper was not yet covered fully in the literature. In [6] the authors consider the 
similar problem and introduce a game theoretical view on it, however they do not consider mobile network operators but rather cloud service providers as players of the game. On the other hand, in $[4,2,9,11]$ the authors consider the problem of competition between MNOs and MVNOs but they work toward solving the radio resource allocation problem in optimal way, which is different from the problem suggested in this paper.

\section{Conclusion}

This paper discussed a competition model on the three-level mobile operator market. The model is presented as a two-staged non-cooperative game. On the first stage that is an allocation game where the players (MVNO) select the MNO market. On the second stage that is a pricing game where they compete between themselves for the consumers. We show that the pricing game is a potential game. It simplifies finding the the equilibrium. Moreover, the payoffs of the players are decreasing functions if the number of MVNOs on the same market is increasing. It yields that in some cases the allocation game on the first stage can be investigated by the methods of the congestion game theory. We analyze in detail the cases of identical MVNOs and non-identical MNOs. A useful characteristic of the MNO markets is developed, that is the ratio of the number of consumers covered by $\mathrm{MNO}$ and the influence of the price on attractability of this operator. All mobile network operators can be ranked on the market using this characteristic.

We make accent in the paper mainly on the analytic investigation of the problem. But the proposed game-theoretical model can be applied in any real mobile network market. The model can be extended to include to the game $\mathrm{MNO}$ as a player. They can also compete in the market determining the price for their resources. This three-stage game is an interesting topic for future study.

\section{Acknowledgment}

The authors acknowledge support from Academy of Finland (grants 296572, 316908 and 311648) and the Center for Industrial Information Technology (CENIIT) project 17.01. Prof. Mazalov is also supported by the Russian Fund for Basic Research (projects 16- 51-55006, 16-01-00183) and Shandong Province DoubleHundred Talent Plan (No. WST2017009).

\section{References}

[1] D. Ardagna, B. Panicucci, and M. Passacantando. A game theoretic formulation of the service provisioning problem in cloud systems. In Proceedings of the 20th International Conference on World Wide Web, WWW '11, pages 177-186, New York, NY, USA, 2011. ACM.

[2] H. Cadre, M. Bouhtou, and B. Tuffin. A pricing model for a mobile network operator sharing limited resource with a mobile virtual network operator. In Proceedings of the 6th International Workshop on Internet Charging 
and Qos Technologies: Network Economics for Next Generation Networks, ICQT '09, pages 24-35, Berlin, Heidelberg, 2009. Springer-Verlag.

[3] A. Kiiski and H. Hammainen. Mobile virtual network operators: Case finland. Technical report, TKK, 2004.

[4] W. Liu, R. Hu, R. Shinkuma, and T. Takahashi. A fair resource sharing mechanism between mobile virtual network operators. IEICE Transactions, 98-B(11):2141-2150, 2015

[5] V. Mazalov. Mathematical Game Theory and Applications. Wiley, 2014.

[6] V. Mazalov, A. Lukyanenko, and S. Luukkainen. Equilibrium in cloud computing market. Perform. Eval., 92(C):40-50, Oct. 2015.

[7] I. Milchtaich. Congestion games with player-specific payoff functions. Games and Economic Behavior, 13(1):111 - 124, 1996.

[8] D. Monderer and L. S. Shapley. Potential games. Games and Economic Behavior, 14(1):124 - 143, 1996.

[9] S. Pattanavichai, N. Jongsawat, and W. Premchaiswadi. A pricing model and sensitivity analysis for MNO's investment decision making in 3G UMTS networks. In Proc. of the Third International Symposium on Electronic Commerce and Security, pages 274-279, July 2010.

[10] R. W. Rosenthal. A class of games possessing pure-strategy Nash equilibria. International Journal of Game Theory, 2(1):65-67, Dec 1973.

[11] S. Zhao, Q. Zhu, and H. Zhu. Demand responsive pricing competition of two MVNOs in dynamic spectrum access. In Proc. of IEEE 12th International Conference on Communication Technology, pages 958-961, Nov 2010. 